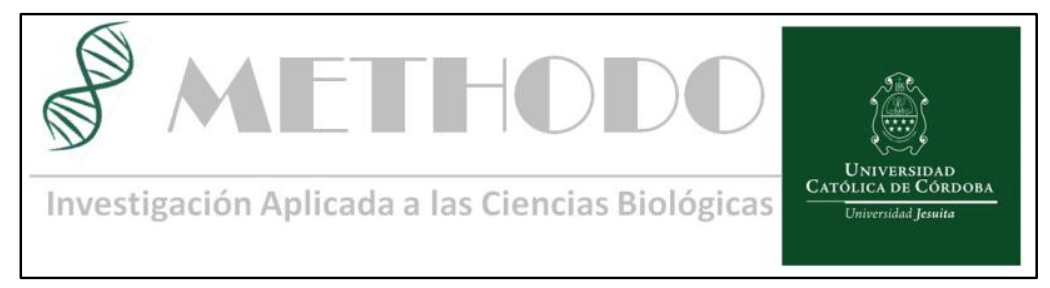

\title{
Ventajas y riesgos del uso de pastas dentales con nanotecnologías
}

\section{Advantages and hazzards of the use of nanotechnology in toothpastes}

\author{
Dra. Marina Rocamundi, Od. ${ }^{1}$ Andrea Lagonero, ${ }^{1}$ Est. Ana Lasca Juncal, ${ }^{1}$ Est. Carolina Pollo, ${ }^{1}$ Est. María \\ Laura Reston Fradejas, ${ }^{1}$ Est. Sol Chiadeh, ${ }^{1}$ Est. Gustavo Ingacio Cerioni, ${ }^{1}$ Est. Daniela Lupica Castro, ${ }^{1}$ \\ Est. Melisa Daher, ${ }^{1}$ Est. Sola Di Genaro ${ }^{1}$
}

${ }^{1}$ Institución: Facultad de Ciencias de la Salud - Carrera de Odontología - Trabajo de Integración Curricular año 2018.

\section{Resumen:}

El presente artículo de actualización analiza las ventajas y riesgos del uso de nanopartículas en pastas dentales. La nanotecnología puede mejorar sus propiedades ayudando al proceso de remineralización del diente, controlar el crecimiento bacteriano o proporcionar minerales para mejorar el control del $\mathrm{pH}$. Con este fin se han agregado: nanohidroxiapatita, nanocalcio, fosfato de calcio, trimetafosfato de sodio, nanopartículas de plata, quitosán, entre otras. Por otro lado, se han descripto algunos efectos nocivos de estas nanotecnologías, lo que nos motiva a intensificar su estudio. Conclusiones: La nano-odontología ha otorgado nuevas herramientas para la atención preventiva de la salud. La toxicidad oral para los nanodentífricos es baja, pero algunos pueden llegar al intestino, y a través de él a la circulación sanguínea y causar disturbios sistémicos. Es necesario profundizar las investigaciones en estos materiales, a fin de mejorar sus efectos beneficiosos, e identificar y eliminar sus riesgos para la salud.

\section{Abstract:}

The present update article analyzes advantages and risks of nanoparticles in relation to toothpastes. Nanotechnology can improve its properties by helping to remineralize the tooth, control bacterial growth or provide minerals to optimize $\mathrm{pH}$ control. For this purpose, anohydroxyapatite, nanocalcium, calcium phosphate, sodium trimetaphosphate, silver nanoparticles, chitosan, among others have been added. On the other hand, some harmful effects of these nanotechnologies have been described, which motivates us to intensify their study. Conclusions: Nano-dentistry has provided new tools for preventive health care. The oral toxicity for nanodentífricos is low, but some can reach the intestine, and through it to the blood circulation and cause systemic disturbances. It is necessary to deepen the investigations in these materials, in order to improve their beneficial effects, and identify and eliminate their health risks.

\section{Introducción:}

El prefijo "nano" proviene del griego y significa "enano". Usualmente se emplea la palabra nanociencia para referirse al estudio de los fenómenos y el manejo de la materia a escala nanométrica $(1 \mathrm{~nm}=0,000000001 \mathrm{~mm})$, mientras que la nanotecnología se encarga del estudio, creación, diseño, síntesis, identificación, manipulación y aplicación de materiales, aparatos y sistemas a través del control de la materia en dimensiones cercanas al intervalo de 1-100 nanómetros, así como de la exploración de fenómenos y propiedades de la materia a dicha escala. $^{1}$ 
La evolución de esta ciencia emergente anticipa un importante rol de la nanotecnología en el campo aplicado a la odontología, denominado nanoodontología. Entre sus principales desafíos se destaca el alcanzar una mejor comprensión de las bases fisiopatológicas de las enfermedades, nuevas estrategias de diagnóstico, y el desarrollo de terapias altamente efectivas. $^{2}$

Existen varias formas de nanomateriales. Las nanopartículas debido a su pequeño tamaño tienen un área de superficie mucho mayor por unidad de masa en comparación con partículas más grandes de la misma composición.3Las nanofibras permiten la invasión y proliferación celular debido a su parecido con la matriz extracelular. Los nanotubos y las nanopartículas mejoran las propiedades mecánicas y químicas del tejido, aumentan la migración de las células y facilitan la regeneración del tejido. Además, las nanofibras y nanopartículas también se usan como un sistema de transporte para agentes bioactivos que promueven la regeneración tisular. ${ }^{4}$ Los compuestos orgánicos pueden ser utilizados para el suministro controlado de antibióticos, factores de crecimiento o incluso drogas oncológicas. ${ }^{5}$

En el presente artículo de actualización, con un enfoque más puntual en la odontología preventiva, vamos a estudiar y analizar los avances de nanopartículas en relación a pastas dentales.

\section{Ventajas}

La nanotecnología puede mejorar las propiedades de las pastas dentales ayudando al proceso de mineralización del diente para detener lesiones de caries y reducir la sensibilidad dental; controlar el crecimiento bacteriano, o proporcionar minerales para mejorar el control del ph., ${ }^{6-7}$ (Figura 1)

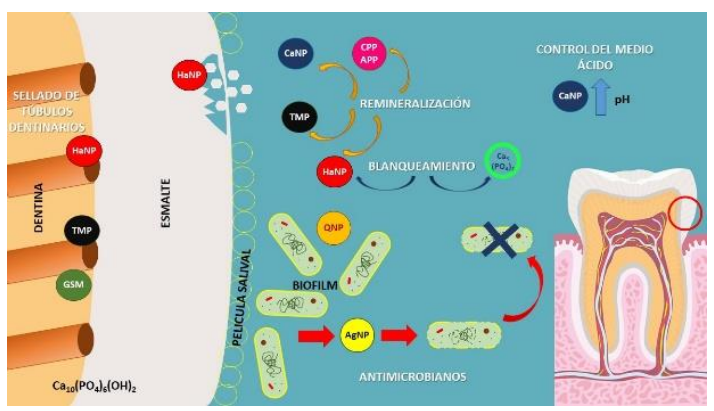

Figura 1. Efectos de los principales nanocomponentes incorporados a pastas dentales: Nanohidroxiapatita (HaNP), Nanoesteras de Fosfato de Calcio

[Ca3(PO4)2], Nanopartículas de Plata (AgNP),

Quitosán (QNP), nanocalcio (CaNP), nanocomplejos CPP-APP, nanopartículas de trimetafosfato de sodio (TMP) y polímeros de extractos de semillas (GSE).

Las nanopartículas de hidroxiapatita resultan muy eficaces en el tratamiento de remineralización del esmalte, de la hipersensibilidad dentinaria y el blanqueamiento dental. ${ }^{8}$ Se integra y restaura la densidad mineral de la superficie del esmalte desmineralizado, proporcionando una superficie más lisa y brillante $\mathrm{y}$, en consecuencia, unos dientes más blancos. ${ }^{9}$ Además, su uso posterior ayuda a mantener el blanqueamiento durante más tiempo y evita la hipersensibilidad postoperatoria. Se ha comprobado que no solo mejora significativamente la microdureza del esmalte, sino que también puede reducir la colonización bacteriana en la superficie del diente. ${ }^{10}$

Sobre la superficie de la dentina, estas nanopartículas obturan los túbulos dentinarios abiertos, presentes en los dientes sensibles, e impiden así la transmisión de estímulos externos a las terminaciones nerviosas de la pulpa y el movimiento del fluído dentinario, y de esta manera evitan el dolor. Éstas forman una capa protectora resistente al lavado desde la primera aplicación.2 Además, esta pasta tiene el más bajo índice de abrasividad, y mejora la resistencia del esmalte al ácido y a la caries. ${ }^{11}$

Por otro lado, se desarrollaron nanoesferas huecas de fosfato de calcio que podrían cargarse con peróxido de carbamida, y utilizarse como partículas portadoras en formulaciones de blanqueamiento dental, sin afectar la liberación de Hidroxiapatita. $^{12}$

Los nanocomplejos CPP-ACP promueven la remineralización. Se adhieren al esmalte, a la película, a la placa y al tejido blando, liberando calcio e iones de fosfato, evitando las caries y erosión en dientes permanentes y primarios. ${ }^{13-14}$

El dentífrico con nanocalcio tiene mayor eficacia en la reparación de caries tempranas, una buena retención en las superficies orales y administración continua de iones de calcio con liberación lenta. Además, aumenta el $\mathrm{pH}$ del líquido que rodea el esmalte de la lesión. ${ }^{15}$

El trimetafosfato de sodio promovió un efecto remineralizante mayor en comparación con una pasta de dientes convencional sobre las lesiones de caries, ${ }^{16}$ y mayor obliteración de los túbulos dentinarios. ${ }^{17}$

Con el objetivo de intentar estabilizar la matriz de colágeno de dentina se han desarrollado polímeros biodegradables provenientes de extractos de semillas (GSE) que reticulan eficazmente al colágeno tipo I, inhiben la actividad de la metaloproteinasa de la matriz y disminuye su tasa de desmineralización. ${ }^{18}$

Además, se han desarrollado nanopartículas para ser utilizadas como portadores biológicos dentro de biofilms en productos de cuidado oral, tales como dentífricos y enjuagues bucales. Su objetivo es inhibir a los microorganismos y bloquear sus mecanismos de variación fenotípica, que les

Revista Methodo: Investigación Aplicada a las Ciencias Biológicas. Universidad Católica de Córdoba. Jacinto Ríos 571 Bo Gral. Paz. X5004FXS. Córdoba. Argentina. Tel.: (54) 351 4517299 / Correo: methodo@ucc.edu.ar / Web: methodo.ucc.edu.ar | ARTICULO ORIGINAL Methodo 2018;3(3):67-72 
permiten adaptarse y sobrevivir en condiciones ambientales adversas.

La plata combinada con flúor inhibe el crecimiento de microorganismos sobre las superficies dentales, alterando la permeabilidad de la membrana, y causando su ruptura. Además, afecta ácidos nucleicos para prevenir el proceso de replicación celular $^{19}$ e inactivas funciones fisiológicas críticas, como la síntesis de la pared celular. ${ }^{20}$

El agregado de quitosán a las pastas tiene un prometedor efecto anticaries y de protección contra la erosión, más fuerte en comparación con la nanohidroxiapatita. ${ }^{21}$

Además, se está trabajando en la creación de dentífricos con nanorobots de una dimensión entre 1-10 $\mu \mathrm{m}$, liberados por enjuagues o dentífricos, que contienen drogas diseñadas genéticamente contra agentes patógenos. Estarían configurados para identificar y destruir otras bacterias patógenas residentes en la cavidad oral que ocasionan halitosis y periodontopatías, pero respetarían las especies de bacterias saprofitas de la microflora. ${ }^{22}$ Las funciones de los nanorobot pueden ser controladas por un nanoordenador que ejecuta las instrucciones preprogramadas en respuesta a los estímulos del sensor local. Estos dentifricorobots podrían patrullar todas las superficies supra y subgingivales al menos una vez al día, metabolizando la materia orgánica atrapada y junto a la realización continua de desbridamiento del cálculo dental. ${ }^{23}$

\section{Riesgos}

Debe ponerse atención y cuidado a los impactos negativos que el desarrollo de nanotecnologías podría implicar para las personas involucradas en las actividades de investigación, innovación, manufactura, transporte, consumo y disposición final de los residuos. Con respecto a las nanociencias y nanotecnologías no se trata sólo de un potencial futuro, en realidad ya está presente en el mercado a través de una gran variedad de productos. Algunos trabajos describen algunos efectos nocivos de estas nanotecnologías, lo que nos motiva a intensificar su estudio. (Figura 2)

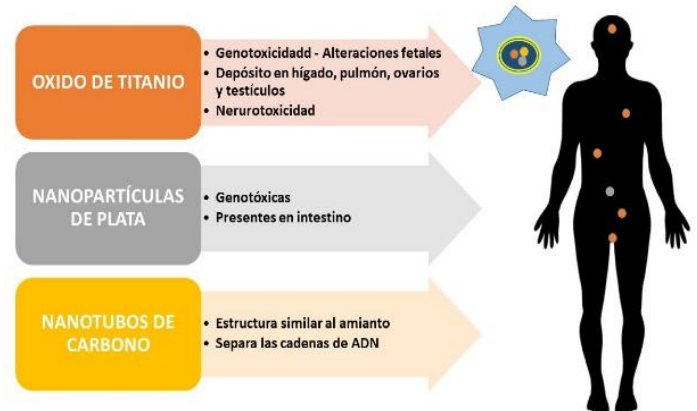

Figura 2. Efectos negativos de nanotecnologías a nivel celular y orgánico. Las principales consecuencias aparecieron al estudiar nanopartículas de óxido de titanio, de plata y nanotubos de carbono.

En cultivos celulares y en modelos animales, se ha demostrado que las nanopartículas incrementan la respuesta celular y presentan características de biomimetismo con los tejidos dentales. ${ }^{24} \mathrm{Al}$ ser del tamaño de componentes naturales, se sugiere que podrían evadir las defensas naturales y causar daño celular.

Los pacientes pueden exponerse a: ingestión accidental o incidental del material, aspiración de aerosoles, toxicidad sistémica de nanopartículas ingeridas o inhaladas, y toxicidad directa a las células de la cavidad oral. La respuesta está relacionada con la dosis y con las propiedades físicas y químicas de las mismas.

Los nanotubos de carbono han sido cuestionados por sus similitudes físicas con las fibras de amianto, y por su capacidad para separar cadenas de ADN, al envolverse alrededor de su molécula. Esto aumenta la preocupación sobre las consecuencias de los mismos entrando en el cuerpo humano. ${ }^{25}$

El dióxido de titanio ( $\mathrm{TiO} 2)$ se aplica comúnmente para mejorar el color blanco y el brillo de los productos alimenticios y cosméticos. Los productos que más contribuyen a su ingesta son la pasta de dientes, dulces, crema de café, productos de panadería fina y salsas ${ }^{26}$ El límite de ingesta de $\mathrm{TiO} 2$ se estima en aproximadamente $1-3 \mathrm{mg} / \mathrm{kg}$ de peso corporal por día.

Estudios recientes con nanopartículas de $\mathrm{TiO} 2$ indican que pueden tener efectos tóxicos.

Debido a que los humanos son especies de larga vida, es importante conocer el impacto de la exposición temprana a nanopartículas con dosis bajas, continuas y a largo plazo. Se observado úteros y fetos más pequeños después de que se les inyectó nanopartículas de $\mathrm{TiO} 2$ por vía intravenosa a ratones preñados, las cuales se encontraron depositadas en la placenta, hígado fetal y cerebro fetal. ${ }^{27}$

Los análisis de genotoxicidad demostraron que deterioraron el ADN e interrumpieron la homeostasis metabólica en el hígado, al tiempo que inducían estrés oxidativo, respuestas inflamatorias y apoptosis en el tejido pulmonar, lo cual puede relacionarse con procesos de carcinogénesis. $^{28}$

En general, la piel sana y mucosas son una mejor barrera que las vías respiratorias, el esófago y el intestino por su mayor espesor, aunque se necesita más investigación para evaluar el efecto sobre la piel dañada o piel estirada. ${ }^{29}$ Además, debemos recordar que las nanomedicinas están siendo diseñadas para cruzar barreras mucosas, y algunas nanopartículas son inmunogénicas e inducirían

Revista Methodo: Investigación Aplicada a las Ciencias Biológicas. Universidad Católica de Córdoba. Jacinto Ríos 571 Bo Gral. Paz. X5004FXS. Córdoba. Argentina. Tel.: (54) 351 4517299 / Correo: methodo@ucc.edu.ar / Web: methodo.ucc.edu.ar | ARTICULO ORIGINAL Methodo 2018;3(3):67-72 
una reacción de hipersensibilidad o inflamación en un paciente vulnerable.

También se evaluaron la incorporación de nanopartículas de $\mathrm{TiO} 2$ de acuerdo su concentración en órganos internos, encontrándose afectados el hígado, los ovarios y los testículos. Se ha demostrado la endocitosis de nanopartículas intactas de $\mathrm{TiO} 2$ por células intestinales en cultivo, a pesar de contener inhibidores del endocitosis, como la nistatina. Se necesita más información sobre las concentraciones de $\mathrm{TiO} 2$ en órganos humanos, y sobre los efectos en el hígado, en los órganos reproductores y fertilidad después de una exposición más prolongada. ${ }^{30}$

La neurotoxicidad del $\mathrm{TiO} 2$ se estudió en ratones, y puede estar asociada con alteraciones significativas de la expresión de genes implicados en estrés oxidativo, apoptosis, memoria y aprendizaje, desarrollo cerebral, metabolismo lipídico, reparación del $\mathrm{ADN}$, transducción de señales, respuesta inmune y respuesta a estímulos. Por lo tanto, estos genes pueden ser biomarcadores potenciales de la toxicidad cerebral causados por la exposición a las nanopartículas de $\mathrm{TiO} 2 .{ }^{31}$ En otro estudio sobre cerebro, las nanopartículas de $\mathrm{TiO} 2$ entraron en las células, y condujeron a la disfunción de la dinámica de los microtúbulos, volviéndolos inestables y acortando el tiempo de vida de las células. ${ }^{32}$

La genotoxicidad de las nanopartículas de plata (AgNP) que contienen algunas pastas dentales, ingeridas por vía oral, depende del material de recubrimiento de superficie de nanopartículas, siendo más perjudiciales las recubiertas con citrato. Una proporción de nanopartículas AgNP siguieron apareciendo en jugo intestinal. Dado que la genotoxicidad es un precursor de la carcinogenicidad, se recomienda continuar su estudio para estos productos. ${ }^{33}$

El riesgo asociado con una exposición al fosfato de calcio nanoparticulado, en dosis que generalmente se aplican en biomedicina, productos para el cuidado de la salud y cosméticos, es muy bajo y muy probablemente no presente en absoluto. ${ }^{34}$

Aunque los beneficios de la nanotecnología son ampliamente publicitados, la discusión sobre los efectos potenciales de su uso generalizado en productos de está solo comenzando. Ambos pioneros, de la nanotecnología y sus oponentes, encuentran extremadamente difícil argumentar su caso debido a la información limitada disponible para apoyar a un lado o el otro. Se sugieren una mejor orientación para los profesionales sobre nanoproductos incorporados con respecto a la seguridad del paciente y la salud laboral, y el etiquetado nanoespecífico de los productos de cuidado personal para pacientes.

\section{Conclusión:}

La nano-odontología ha otorgado nuevas herramientas para la atención preventiva de la salud, con pastas dentales antimicrobianas y con propiedades reconstituyentes para el esmalte.

Los datos hasta ahora indican que la toxicidad oral para los nanodentífricos es baja, pero algunos pueden llegar al intestino, y a través de él a la circulación sanguínea y causar disturbios sistémicos.

Es necesario profundizar las investigaciones en estos materiales, a fin de mejorar sus efectos beneficiosos, e identificar y eliminar sus riesgos para la salud.

\section{Bibliografía}

1. Martínez HR, Abdala HM, Treviño E, Garza $\mathrm{G}$ et al. Aplicación de la nanotecnología en odontología: Nano-odontología. Rev.CES Odont.2011;24(2)87-91.

2. Cantin M, Vilos OC, Suaz GI. Nanoodontologia: El futuro de la Odontología basada en sistemas nanotecnologicos. Int. J. Odontostomat 2010;4(2):127-132.

3. Bhardwaj A, Bhardwaj A, MisuriyaA, Maroli $S$ et al. Nanotechnology in dentistry: Present and future. $\mathbf{J}$ of Int Oral Health 2014; 6(1):121-126.

4. Li G, Zhou T, Lin S, Shi S et al. Nanomaterials for Craniofacial and Dental Tissue Engineering. J Dent Res. 2017 Jul;96(7):725732 .

5. Virlan MJR, Miricescu D, Radulescu R, Sabliov CM et al. Organic Nanomaterials and Their Applications in the Treatment of Oral Diseases. Molecules 2016, 21, 207; doi:10.3390/molecules21020207

6. Besinis A, De Peralta T1, Tredwin CJ1, Handy RD. Review of nanomaterials in dentistry: interactions with the oral microenvironment, clinicalapplicatio ns, hazards, and benefits. ACS Nano. 2015 Mar 24;9(3):2255-89.

7. Cheng L, Zhang K, Weir MD, Melo MA et al. Nanotechnology strategies for antibacterial and remineralizing composites and adhesives to tackle dental caries. Nanomedicine 2015;10(4):627-41

8. Hill RG, Gillam DG, Chen X. The ability of a nano hydroxyapatite toothpaste and oral rinse containing fluoride to protect enamel during 
an acid challenge using 19F solid state NMR spectroscopy. Materials Letters 2015;156: 69-71.

9. Esteves-Oliveira M, Santos NM, MeyerLueckel H, Wierichs RJ et al. Cariespreventive effect of anti-erosive and nanohydroxyapatite-containing toothpastes in vitro. Clin Oral Investig. 2017 Jan;21(1):291300 .

10. Mielczarek A, Michalik J. The effect of nanohydroxyapatite toothpaste on enamel surface remineralization. An in vitrostudy. Am J Dent 2014 Dec;27(6):287-90.

11. Makeeva IM, Polyakova MA, Avdeenko OE, Paramonov YO et al. Effect of long term application of toothpaste Apadent Total Care Medical nano-hydroxyapatite. Stomatologiia (Mosk) 2016;95(4):34-36.

12. Mellgren T, Qin T, Öhman-Mägi C, Zhang Y et al. Calcium Phosphate Microspheres as a Delivery Vehicle for Tooth-Bleaching Agents. J Dent Res 2018, 97(3):283-288.

13. Rai N, Sandhu M, Sachdev V, Sharma R. Evaluation of Remineralization Potential of Beverages modified with Casein Phosphopeptide-Amorphous Calcium Phosphate on Primary and Permanent Enamel: A Laser Profiler Study. Int J of Clin Pediatric Dentistry, 2018;11(1):7-12

14. Souza BM, Comar LP, Vertuan M, Fernandes Neto $C$ et al. Effect of an Experimental Paste with Hydroxyapatite Nanoparticles and Fluoride on Dental Demineralisation and Remineralisation in situ. Caries Res 2015; 49:499-507

15. Rahardjo A, Nugraheni DDT, Humaira G, Adiatman M Et al. Efficacy of Toothpaste Containing Nano Calcium in Dentin Remineralization. Makara J Health Res 2015,19(2):43-47.

16. Danelon M, PelimPessan J, SouzaNeto FN, Rodrigues de Camargo $\mathrm{E}$ et al. Effect of toothpaste with nano-sized trimetaphosphate on dental caries: In situ study. J Dent 2015 43(7):806-813.

17. Favretto CO, Delbem ACB, Moraes JCS, Camargo ER et al. Dentinal tubule obliteration using toothpastes containing sodium trimetaphosphatemicroparticles or nanoparticles. Clin Oral Investig. 2018 Feb 20. doi: 10.1007/s00784-018-2384-3.

18. Fawzy AS, Priyadarshini BM, Selvan ST, Lu TB et al. Proanthocyanidins-Loaded
Nanoparticles Enhance Dentin Degradation Resistance. J Dent Res 2017;96(7):780-789

19. Teixeira JA, Silva AVCE, Dos Santos Júnior VE, de Melo Júnior PC et al. Effects of a New Nano-Silver Fluoride Containing Dentifrice on Demineralization of Enamel and Streptococcus mutans Adhesion and Acidogenicity. Int J Dent 2018 May 8:1351925.

20. Bhardwaj SB, Mehta M, Gauba K. Nanotechnology: Role in dental biofilms. Indian J Dent Res 2009; 20:511-3.

21. Chávez de Paz LE, Resin A, Howard KA, Sutherland DS et al. Antimicrobial Effect of Chitosan Nanoparticles on Streptococcus mutans Biofilms. Appl Environ Microbiol 2011; June:3892-5

22. Mishra S. Nanotechnology in medicine. Indian Heart J. 2016 May-Jun;68(3):437-9.

23. Khurshid Z, Zafar M, Qasim S, Shahab S Et al. Advances in Nanotechnology for Restorative Dentistry. Materials 2015, 8, 717731.

24. De la Fuente Hernández J, Álvarez Pérez MA, Sifuentes Valenzuela MC. Uso de nuevas tecnologías en odontología. Revista Odontológica Mexicana 2011;15 (3): 157-162

25. Sahoo SK, Parveen S, Panda JJ. The present and future of nanotechnology in human health care. Nanomedicine. 2007 Mar;3(1):20-31.

26. Rompelberg $\mathrm{C}$, Heringa MB, van Donkersgoed G, Drijvers J Et al. Oral intake of added titanium dioxide and its nanofraction from food products, food supplements and toothpaste by the Dutch population. Nanotoxicology. 2016;10(10):1404-1414.

27. World Organization Health. Nanotechnology and human health: Scientific evidence and risk governance. Report of the WHO expert meeting 10-11. December 2012, Bonn, Germany. Copenhagen, WHO Regional Office for Europe, 2013.

28. Li Y, Yan J, Ding W, Chen $Y$ et al. Genotoxicity and gene expression analyses of liver and lung tissues of mice treated with titanium dioxide nanoparticles. Mutagenesis 2017; 32:33-46.

29. Bernauer U, Bodin L, Celleno L, Chaudhry Q et al. SCCS OPINION ON Titanium Dioxide (nano form) as UV-Filter in spraysSCCS/1583/17 Final versión. 2018. <hal01695521> 
30. Heringa MB, Geraets L, Van Eijkeren JC, Vandebriel RJ et al. Risk assessment of titanium dioxide nanoparticles via oral exposure, including toxicokinetic considerations. Nanotoxicology. 2016;10(10):1515-25.

31. Ze Y, Hu R, Wang X, Sang X, Ze X, Li B, et al. Neurotoxicity and gene expressed profile in brain injured mice caused by exposure to titanium dioxide nanoparticles. J Biomed Mater Res A 2014;102(2):470-8.

32. Mao Z, Xu B, Ji X, Zhou K Et al. Titanium dioxide nanoparticles alter cellular morphology via disturbing the microtubule dynamics. Nanoscale 2015. DOI: $10.1039 / \mathrm{c} 5 \mathrm{nr} 01448 \mathrm{~d}$

33. Nallanthighal S, Chan C, Bharalid DJ, Mousad SA et al. Particle coatings but not silver ions mediate genotoxicity of ingested silver nanoparticles in a mouse model. NanoImpact 2017; 5:92-100.

34. Epple M. Review of potential health risks associated with nanoscopic calcium phosphate.

Acta

\section{Palabras claves:}

NANO-ODONTOLOGÍA

NANODENTÍFRICOS - NANOTOXICIDAD.

\section{Keywords:}

NANOODONTOLOGY - NANODENTIFRICES NANOTOXICITY.

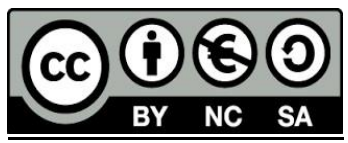

